\title{
LAGUERRE ARC LENGTH FROM DISTANCE FUNCTIONS*
}

\author{
DAVID E. BARRETT ${ }^{\dagger}$ AND MICHAEL BOLT ${ }^{\ddagger}$
}

\begin{abstract}
For the Laguerre geometry in the dual plane, invariant arc length is shown to arise naturally through the use of a pair of distance functions. These distances are useful for identifying equivalence classes of curves, within which the extremal curves are proved to be strict maximizers of Laguerre arc length among three-times differentiable curves of constant signature in a prescribed isotopy class. For smoother curves, it is shown that Laguerre curvature determines the distortion of the distance functions. These results extend existing work for the Möbius geometry in the complex plane.
\end{abstract}

Key words. Distance function, dual number, Laguerre arc length, Laguerre geometry.

AMS subject classifications. Primary 51B15; Secondary 53A35, 58E35

1. Introduction. Laguerre geometry classically refers to a geometric study of oriented circles and lines in two dimensions, or else oriented spheres and hyperplanes in higher dimensions, where the Laguerre transformations are bijective maps that preserve oriented contact. These transformations include the rigid motions and similarities, as well as a unique class of maps, the dilatations, that add a constant to each signed radius and therefore fail to preserve points. The theory originated with Laguerre's work on sphere geometry in the 1850's and was subsequently developed in the early twentieth century, for instance, by Blaschke, Klein, and Liebmann $[2,10,17,20]$.

Recently there has been renewed interest in the subject because of its applications to computational geometry and computer aided geometric design. For example, when combined with the Hough transform, three-dimensional Laguerre geometry can be used for detecting shapes such as planes, spheres, cones, cylinders, and developable surfaces. The geometry can also be used for the design and manipulation of certain surfaces, including canal surfaces generated by a one parameter family of spheres. See, for instance, work by Peternell, Pottmann, and Steiner [15, 16, 18].

This paper is concerned with a model of two-dimensional Laguerre geometry that is expressed using dual numbers. Here, the extended dual plane $\widehat{\mathbb{D}}$ consists of the finite dual numbers $\mathbb{D}=\left\{x+j y: x, y \in \mathbb{R}, j^{2}=0\right\}$ and a line at infinity $L_{\infty}=\left\{(\alpha j)^{-1}: \alpha \in\right.$ $\mathbb{R}\}$. In this model, the Laguerre transformations are the linear fractional and antilinear fractional transformations. The situation is therefore analogous to the study of Möbius transformations over $\mathbb{C}$. Indeed, Laguerre transformations can be interpreted as the parabolic case of a general Möbius transformation. See, for instance, the recent survey by Kisil [7].

The motivation for the present article arises from this connection with complex analysis and a primary goal will be to establish the following results, which are analogous to results the second author proved for the complex plane [5].

THEOREM 1. Consider curves (with endpoints) of class $C^{3}$ in $\widehat{\mathbb{D}}$ that can be

\footnotetext{
*Received October 25, 2009; accepted for publication March 3, 2010.

${ }^{\dagger}$ Department of Mathematics, University of Michigan, 530 Church Street, Ann Arbor, Michigan 48109-1043, USA (barrett@umich.edu). The first author was supported in part by the National Science Foundation under Grant No. DMS-0901205.

${ }^{\ddagger}$ Department of Mathematics and Statistics, Calvin College, 1740 Knollcrest Circle SE, Grand Rapids, Michigan 49546-4403, USA (mbolt@calvin.edu). The second author was supported by the National Science Foundation under Grant No. DMS-0702939 and by Calvin College through a Calvin Research Fellowship.
} 
locally represented as a graph $z=x+j y$ with $d^{3} y / d x^{3} \neq 0$. Among the curves that connect the same fixed osculating endpoint parabolas and belong to the same isotopy class, there is exactly one extremal arc. This extremal arc uniquely maximizes the Laguerre arc length among these curves.

This leads naturally to the following more geometric result.

TheOREM 2. Consider curves (with endpoints) of class $C^{3}$ in $\widehat{\mathbb{D}}$ that can be locally represented as a graph $z=x+j y$ with $d^{3} y / d x^{3} \neq 0$. Their Laguerre class is a quadruple $\left(\epsilon, n, \theta_{\mathrm{lag}}, \delta_{\mathrm{lag}}\right)$ where $\epsilon=\operatorname{sgn}\left(d^{3} y / d x^{3}\right)$ and $n$ identifies the isotopy. In each such class, there is exactly one extremal arc up to (direct) Laguerre transformation and this extremal arc uniquely maximizes the Laguerre arc length.

Liebmann proved that the Laguerre extremals, i.e., the curves that are stationary with respect to Laguerre arc length, are the curves with constant Laguerre curvature [10]. Our results show that these curves maximize the Laguerre arc length. As mentioned, the situation is analogous to the case of Möbius invariant geometry over $\mathbb{C}$. It is also analogous to the case of pseudoaffine geometry in $\mathbb{R}^{2}$, where the parabolic arcs have constant (zero) affine curvature, and they uniquely maximize the affine arc length. See Blaschke [3, p.40], for instance.

More generally, in this article we establish a method for studying Laguerre geometry through the use of distance functions, $\theta_{\text {lag }}$ and $\delta_{\text {lag }}$. These distances are defined on the space of vertical parabolas and nonvertical lines in the dual plane; they are analogous to the Kerzman-Stein and Coxeter distances defined on the space of circles and lines in the complex plane. (Their appearance in Theorem 2 refers to their values on the osculating parabolas at the endpoints.) In earlier work, the authors used these distances to provide estimates for certain Möbius invariant operators that include the Cauchy transform and Kerzman-Stein operator [1]. In $\S 6$ we pursue analogous results for invariant operators in $\mathbb{D}$.

Throughout the article, there are new results interspersed with existing ones, so to avoid disruptions in the presentation, we have chosen to collect the relevant citations in a final section. (Often the citations require some sorting out of different terminologies and normalizations.) Hopefully, by postponing the comments, we are able to reduce the amount of confusion without neglecting proper attribution.

2. Vertical parabolas and Laguerre transformations. We begin by describing the Laguerre geometry of the dual plane and indicate the special role played by the space of vertical parabolas [21].

A dual number $z \in \mathbb{D}$ is a formal expression $z=x+j y$ where $x, y \in \mathbb{R}$ and $j^{2}=0$. These numbers form a commutative algebra over $\mathbb{R}$ where addition and multiplication are done in the obvious way. One identifies dual numbers with points in the real plane via $x+j y \in \mathbb{D} \leftrightarrow(x, y) \in \mathbb{R}^{2}$. The coordinates of $z=x+j y$ are the real part and dual part, respectively. So $x=\operatorname{Real}(x+j y)$ and $y=\operatorname{Dual}(x+j y)$. Geometrically, addition is done by adding position vectors. Multiplication is done by multiplying the real parts and adding the slopes of position vectors.

The direct and indirect Laguerre transformations are the linear fractional and antilinear fractional transformations,

$$
\mu(z)=\frac{a z+b}{c z+d} \quad \text { and } \quad \mu(z)=\frac{a \bar{z}+b}{c \bar{z}+d},
$$

respectively, where $a, b, c, d \in \mathbb{D}$ and $a d-b c= \pm 1[13$, p.390]. The condition $a d-b c=$ 
\pm 1 acts as a normalization and has no effect on the transformation itself. It is just necessary that $\operatorname{Real}(a d-b c) \neq 0$ or else $\mu$ maps $\widehat{\mathbb{D}}$ to a line or point.

Like the Möbius transformations over $\mathbb{C}$, the direct Laguerre transformations form a group that is generated by elements of the following three types:

i) [translation] $: \mu(z)=z+b$ for $b \in \mathbb{D}$

ii) [rotation and dilation] : $\mu(z)=a z$ for $a \in \mathbb{D}$, $\operatorname{Real}(a) \neq 0$

iii) [inversion] : $\mu(z)=1 / z$.

These transformations preserve angles (measured as differences in slope); the indirect Laguerre transformations reverse angles.

Both the direct and indirect transformations preserve the space $X$ of vertical parabolas and nonvertical lines. By a vertical parabola we mean that the axis of symmetry is vertical. The vertical parabolas and nonvertical lines can be described collectively as the graphs $y=a x^{2}+b x+c$ for $a, b, c \in \mathbb{R}$. This fact can be verified for the direct transformations by considering the three types of transformations mentioned above. It then follows for indirect transformations, too, since obviously the conjugation $z \rightarrow \bar{z}=x-j y$ preserves the space.

By using stereographic projection, the extended dual plane $\widehat{\mathbb{D}}=\mathbb{D} \cup L_{\infty}$ can be viewed as an infinite cylinder as illustrated in Figure 1. (Laguerre transformations that do not arise as translations or similarities are induced, nonetheless, by affine symmetries of the cylinder.) In this model, the set $L_{\infty}=\left\{(\alpha j)^{-1}: \alpha \in \mathbb{R}\right\}$ corre-

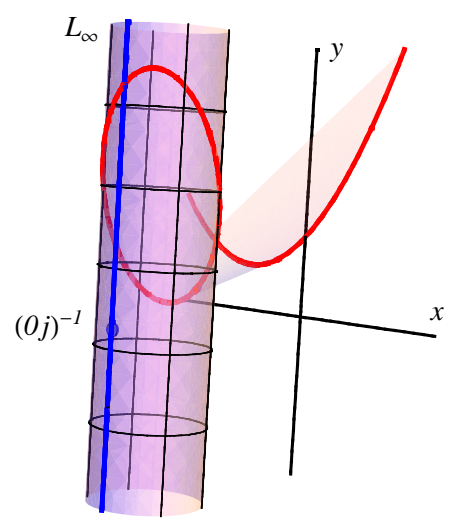

FIG. 1. The representation of the dual plane on the Blaschke cylinder obtained via stereographic projection.

sponds with a line of points at infinity. By using the transformation $\mu(z)=1 / z$, one can see that the parabola $y=a x^{2}+b x+c$ intersects $L_{\infty}$ at the point $-1 /(a j)$, where $a, b, c \in \mathbb{R}$. In particular, the nonvertical lines are the parabolas that intersect $L_{\infty}$ at $1 /(0 j)$.

The space $X$ can be identified with $\mathbb{R}^{3}$ in an obvious way: the parabola $y=$ $a x^{2}+b x+c$ corresponds with the point $(a, b, c) \in \mathbb{R}^{3}$. We use as a pseudodistance $\Delta: X \times X \rightarrow \mathbb{R}$ defined via $\Delta\left(a_{1}, b_{1}, c_{1} ; a_{2}, b_{2}, c_{2}\right)=\left(b_{1}-b_{2}\right)^{2}-4\left(a_{1}-a_{2}\right)\left(c_{1}-c_{2}\right)$. This is not a distance in the usual sense. For instance, it vanishes for parabolas that are tangent to each other. It also vanishes for parabolas that are vertical translates of each other - such parabolas have a unique intersection point on $L_{\infty}$. 
The pseudodistance has an interesting geometric meaning. For intersecting parabolas, $\sqrt{\Delta}$ is the difference in slope at the point of intersection, and can be interpreted as the angle of intersection of the parabolas. For nonintersecting parabolas, $\Delta$ is nonpositive, and $\sqrt{-\Delta}$ can be interpreted as the imaginary angle of intersection.

3. Distance functions and Laguerre arc length. Let $X^{*}$ be the space of pointed vertical parabolas. We describe two invariant distances on $X^{*}$.

(From now on, 'parabola' or 'vertical parabola' will refer to an element of $X$, that is, either a vertical parabola or nonvertical line.)

Suppose $\mathbf{p}_{1}^{*}, \mathbf{p}_{2}^{*} \in X^{*}$ are parabolas that don't intersect as subsets of $\widehat{\mathbb{D}}$. Their Kerzman-Stein distance is defined to be the angle of intersection of two new parabolas constructed using only the corresponding support line elements [1]. The parabola $\mathbf{p}_{12}$ is tangent to the first line element at the first point and passes through the second point; the parabola $\mathbf{p}_{21}$ is tangent to the second line element at the second point and passes through the first point. Clearly these parabolas intersect, and so we define $\theta_{\operatorname{lag}}\left(\mathbf{p}_{1}^{*}, \mathbf{p}_{2}^{*}\right)=\sqrt{\Delta\left(\mathbf{p}_{12}, \mathbf{p}_{21}\right)}$.

The Coxeter distance between the parabolas $\mathbf{p}_{1}^{*}, \mathbf{p}_{2}^{*}$ is gotten by taking the imaginary angle of intersection between the corresponding unpointed parabolas [6]. If the unpointed parabolas are $\mathbf{p}_{1}, \mathbf{p}_{2}$, then $\delta_{\operatorname{lag}}\left(\mathbf{p}_{1}^{*}, \mathbf{p}_{2}^{*}\right)=\sqrt{-\Delta\left(\mathbf{p}_{1}, \mathbf{p}_{2}\right)}$.

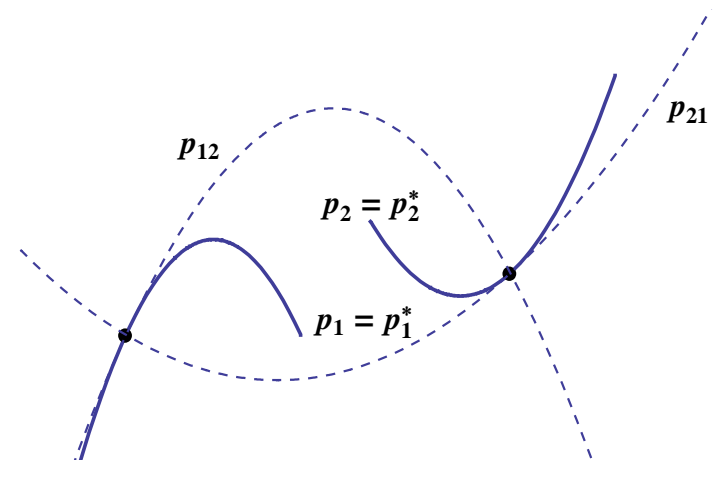

FIG. 2. The Kerzman-Stein distance between nonintersecting pointed parabolas is $\theta_{\operatorname{lag}}\left(\mathbf{p}_{1}^{*}, \mathbf{p}_{2}^{*}\right)=$ $\sqrt{\Delta\left(\mathbf{p}_{12}, \mathbf{p}_{21}\right)}$ and the Coxeter distance is $\delta_{\mathrm{lag}}\left(\mathbf{p}_{1}^{*}, \mathbf{p}_{2}^{*}\right)=\sqrt{-\Delta\left(\mathbf{p}_{1}, \mathbf{p}_{2}\right)}$.

In order to define arc length, let $\gamma \subset \mathbb{D}$ be a twice differentiable curve, and let $T(\gamma) \subset X^{*}$ be the family of osculating pointed vertical parabolas. There is a natural ordering on $T(\gamma)$ that is determined by the orientation of $\gamma$. Let $P$ be a partition of $T(\gamma)$. This consists of a finite subset of $T(\gamma)$ that includes the osculating endpoint parabolas. Figure 3 shows an example of one such partition $P \subset T(\gamma)$.

If one restricts to smooth curves $z=x+j y$ for which $d^{3} y / d x^{3} \neq 0$, then one may be sure that the osculating parabolas are non-intersecting. So to simplify matters, we from now on make this assumption. We also denote a curve's signature by $\epsilon=$ $\operatorname{sgn}\left(d^{3} y / d x^{3}\right)= \pm 1$ and mention that the signature is preserved by a direct Laguerre transformation. The signature is reversed by an indirect transformation.

By taking limits of Riemann sums, we arrive at two possible formulations of 


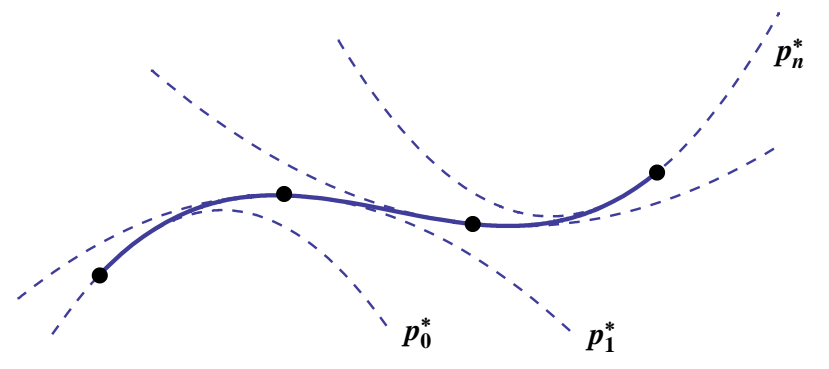

FIG. 3. For an oriented curve, the elements in a partition of osculating parabolas have a natural ordering $\mathbf{p}_{0}^{*}<\mathbf{p}_{1}^{*}<\cdots<\mathbf{p}_{n}^{*}$.

Laguerre arc length:

$$
\begin{aligned}
& \liminf _{P \subset T(\gamma),\|P\| \rightarrow 0} \sum_{i} \sqrt{6} \theta_{\operatorname{lag}}\left(\mathbf{p}_{i}^{*}, \mathbf{p}_{i+1}^{*}\right)^{1 / 2} \\
& \liminf _{P \subset T(\gamma),\|P\| \rightarrow 0} \sum_{i} \sqrt[4]{12} \delta_{\operatorname{lag}}\left(\mathbf{p}_{i}^{*}, \mathbf{p}_{i+1}^{*}\right)^{1 / 2}
\end{aligned}
$$

The scaling factors are chosen so that the formulations agree with each other and with a third formulation $[2,8,10,13]$ obtained using the invariance of the Schwarzian,

$$
\{z ; t\} \stackrel{\text { def }}{=}\left(\frac{z^{\prime \prime}}{z^{\prime}}\right)^{\prime}-\frac{1}{2}\left(\frac{z^{\prime \prime}}{z^{\prime}}\right)^{2} .
$$

Lemma 1. For a three-times differentiable curve $\gamma \subset \mathbb{D}$ that is parameterized by $z=z(t)$ for $t \in\left[t_{0}, t_{1}\right]$, the quantity $\int_{t_{0}}^{t_{1}} \sqrt{\epsilon \operatorname{Dual}\{z ; t\}} d t$ is Laguerre invariant. (It is also independent of the parameterization.)

Proof. The usual argument for the Schwarzian shows that for a direct Laguerre transformation $\mu$, one has $\{\mu \circ z ; t\}=\{z ; t\}$. Moreover, under change of parameter $t^{\prime}=t^{\prime}(t)$ there is the formula for the change in Schwarzian,

$$
\{z ; t\}=\left(\frac{d t^{\prime}}{d t}\right)^{2}\left\{z ; t^{\prime}\right\}+\left\{t^{\prime}, t\right\}
$$

Since $\left\{t^{\prime}, t\right\}$ is real, one obtains from this the invariant one-form $\sqrt{\epsilon \operatorname{Dual}\{z ; t\}} d t$.

The essence of the following proposition is that the three formulations of Laguerre arc length agree with one another.

Proposition 1. For a three-times differentiable curve $\gamma$ parameterized by $z=x+$ jy with $d^{3} y / d x^{3} \neq 0$, the following formulations of Laguerre arc length are equivalent:

i) $\int_{t_{0}}^{t_{1}} \sqrt{\epsilon \operatorname{Dual}\{z ; t\}} d t$

ii) $\liminf _{P \subset T(\gamma),\|P\| \rightarrow 0} \sum_{i} \sqrt{6} \theta_{\operatorname{lag}}\left(\mathbf{p}_{i}^{*}, \mathbf{p}_{i+1}^{*}\right)^{1 / 2}$

iii) $\liminf _{P \subset T(\gamma),\|P\| \rightarrow 0} \sum_{i} \sqrt[4]{12} \delta_{\operatorname{lag}}\left(\mathbf{p}_{i}^{*}, \mathbf{p}_{i+1}^{*}\right)^{1 / 2}$

iv) $\int_{\gamma} \sqrt{\epsilon d^{3} y / d x^{3}} d x$ 
Proof. We show individually that each definition i), ii), and iii) agrees with definition iv).

i) $\int_{t_{0}}^{t_{1}} \sqrt{\epsilon \operatorname{Dual}\{z ; t\}} d t$

Since the quantity is independent of parameterization, we use the graph parameter, $z(x)=x+j y(x)$. Then $z^{\prime}(x)=1+j y^{\prime}(x)$ and $z^{\prime \prime}(x)=j y^{\prime \prime}(x)$, so

$$
\{z ; x\}=\left(\frac{z^{\prime \prime}}{z^{\prime}}\right)^{\prime}-\frac{1}{2}\left(\frac{z^{\prime \prime}}{z^{\prime}}\right)^{2}=\left(\frac{j y^{\prime \prime}}{1+j y^{\prime}}\right)^{\prime}-\frac{1}{2}\left(\frac{j y^{\prime \prime}}{1+j y^{\prime}}\right)^{2}=j y^{\prime \prime \prime} .
$$

It follows that $\int_{t_{0}}^{t_{1}} \sqrt{\epsilon \operatorname{Dual}\{z ; t\}} d t=\int_{t_{0}}^{t_{1}} \sqrt{\epsilon \operatorname{Dual}\{z ; x\}} d x=\int_{\gamma} \sqrt{\epsilon d^{3} y / d x^{3}} d x$.

ii) $\liminf _{P \subset T(\gamma),\|P\| \rightarrow 0} \sum_{i} \sqrt{6} \theta_{\operatorname{lag}}\left(\mathbf{p}_{i}^{*}, \mathbf{p}_{i+1}^{*}\right)^{1 / 2}$

Let $P$ be a partition of $T(\gamma)$ and let $\mathbf{p}_{i}^{*} \in P$ be the $i$ th parabola that osculates $\gamma$ at $z\left(x_{i}\right)$. (Subscripts index the elements of the partition; primes on $y$ indicate derivatives with respect to the graph parameter $x$.) The constructed parabola tangent at $z\left(x_{i}\right)$ and through $z\left(x_{i+1}\right)$ can then be expressed as

$$
\mathbf{p}_{i, i+1}: \quad y=y_{i}+y_{i}^{\prime}\left(x-x_{i}\right)+\frac{y_{i+1}-y_{i}-y_{i}^{\prime}\left(\Delta x_{i}\right)}{\left(\Delta x_{i}\right)^{2}}\left(x-x_{i}\right)^{2}
$$

where $\Delta x_{i}=x_{i+1}-x_{i}$. By interchanging $i$ and $i+1$ one finds a similar expression for the parabola $\mathbf{p}_{i+1, i}$ tangent at $z\left(x_{i+1}\right)$ and through $z\left(x_{i}\right)$,

$$
\mathbf{p}_{i+1, i}: \quad y=y_{i+1}+y_{i+1}^{\prime}\left(x-x_{i+1}\right)+\frac{y_{i}-y_{i+1}+y_{i+1}^{\prime}\left(\Delta x_{i}\right)}{\left(\Delta x_{i}\right)^{2}}\left(x-x_{i+1}\right)^{2} .
$$

Expanding at $x_{i}$ and utilizing a routine computation (done with Mathematica),

$$
\theta_{\operatorname{lag}}\left(\mathbf{p}_{i}^{*}, \mathbf{p}_{i+1}^{*}\right)=\sqrt{\Delta\left(\mathbf{p}_{i, i+1}, \mathbf{p}_{i+1, i}\right)}=\frac{\epsilon y_{i}^{\prime \prime \prime}}{6}\left(\Delta x_{i}\right)^{2}+o\left(\left(\Delta x_{i}\right)^{2}\right) .
$$

It follows that $\liminf \operatorname{i\subset T}_{P \subset T(\gamma),\|P\| \rightarrow 0} \sum_{i} \sqrt{6} \theta_{\operatorname{lag}}\left(\mathbf{p}_{i}^{*}, \mathbf{p}_{i+1}^{*}\right)^{1 / 2}=\int_{\gamma} \sqrt{\epsilon d^{3} y / d x^{3}} d x$.

iii) $\liminf \operatorname{icT}_{P \subset T),\|P\| \rightarrow 0} \sum_{i} \sqrt[4]{12} \delta_{\operatorname{lag}}\left(\mathbf{p}_{i}^{*}, \mathbf{p}_{i+1}^{*}\right)^{1 / 2}$

Again let $P$ be a partition of $T(\gamma)$ and let $\mathbf{p}_{i}^{*} \in P$ be the $i$ th parabola that osculates $\gamma$ at $z\left(x_{i}\right)$. The corresponding unpointed parabola can be expressed as

$$
\mathbf{p}_{i}: y=y_{i}+y_{i}^{\prime}\left(x-x_{i}\right)+\frac{1}{2} y_{i}^{\prime \prime}\left(x-x_{i}\right)^{2} .
$$

(Still, subscripts index the elements of the partition; primes on $y$ indicate derivatives with respect to the graph parameter $x$.) By replacing $i$ with $i+1$ one finds the expression for the parabola at $z\left(x_{i+1}\right)$,

$$
\mathbf{p}_{i+1}: y=y_{i+1}+y_{i+1}^{\prime}\left(x-x_{i+1}\right)+\frac{1}{2} y_{i+1}^{\prime \prime}\left(x-x_{i+1}\right)^{2} .
$$

Expanding at $x_{i}$ and again utilizing a routine computation,

$$
\delta_{\operatorname{lag}}\left(\mathbf{p}_{i}^{*}, \mathbf{p}_{i+1}^{*}\right)=\sqrt{-\Delta\left(\mathbf{p}_{i}, \mathbf{p}_{i+1}\right)}=\frac{\epsilon y_{i}^{\prime \prime \prime}}{\sqrt{12}}\left(\Delta x_{i}\right)^{2}+o\left(\left(\Delta x_{i}\right)^{2}\right) .
$$

It follows that $\liminf \operatorname{in}_{P \subset T(\gamma),\|P\| \rightarrow 0} \sum_{i} \sqrt[4]{12} \delta_{\operatorname{lag}}\left(\mathbf{p}_{i}^{*}, \mathbf{p}_{i+1}^{*}\right)^{1 / 2}=\int_{\gamma} \sqrt{\epsilon d^{3} y / d x^{3}} d x$ 
4. Stationary curves. The following curves and their direct Laguerre images are the extremals of two-dimensional Laguerre geometry in the dual plane [13, p.402403]:

$$
\begin{aligned}
& z\left(s^{\operatorname{lag}}\right)=\tan \left[s^{\operatorname{lag}} \sqrt{\kappa^{\operatorname{lag} / 2}}\left(1+\epsilon j /\left(2 \kappa^{\operatorname{lag}}\right)\right)\right] \\
& z\left(s^{\operatorname{lag}}\right)=s^{\operatorname{lag}}+\epsilon j\left(s^{\operatorname{lag}}\right)^{3} / 6 \\
& z\left(s^{\operatorname{lag}}\right)=\tanh \left[s^{\operatorname{lag}} \sqrt{-\kappa^{\operatorname{lag}} / 2}\left(1+\epsilon j /\left(2 \kappa^{\operatorname{lag}}\right)\right)\right] .
\end{aligned}
$$

These parameterizations are expressed in terms of the Laguerre arc length parameter,

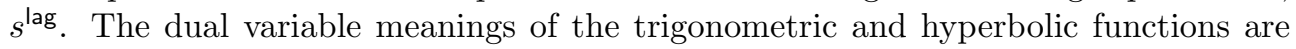
gotten using power series and will become apparent in what follows.

First, the curves are extremal in the sense that they have constant Laguerre curvature $\kappa^{\text {lag }}$ where $\kappa^{\text {lag }}$ is positive, zero, or negative, respectively.

Second, as proved by Liebmann, these curves are extremal in the sense that they are stationary with respect to Laguerre arc length.

Finally, in the next section we prove these curves are maximizers of Laguerre arc length. The three kinds of extremal curves are illustrated in Figure 4.
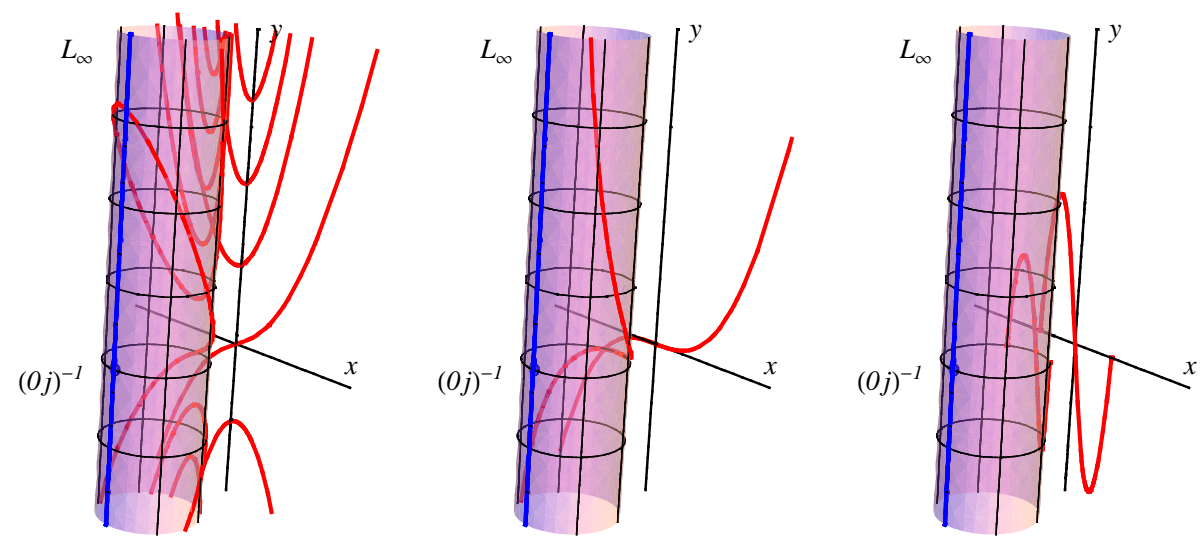

FIG. 4. Extremals on the Blaschke cylinder with $\kappa^{\text {lag }}=1 ; 0 ;-.08$.

For the case $\kappa^{\text {lag }}>0$, the curve crosses the line at infinity each time $s^{\text {lag }} \sqrt{\kappa^{\text {lag }} / 2}$ passes an odd multiple of $\pi / 2$. This is seen more easily when the parameterizations are expanded into their real and dual parts:

$$
\begin{aligned}
& z\left(s^{\operatorname{lag}}\right)=\tan \left[s^{\operatorname{lag}} \sqrt{\kappa^{\operatorname{lag}} / 2}\right]+\frac{\epsilon j s^{\operatorname{lag}}}{2 \sqrt{2 \kappa^{\operatorname{lag}}}} \sec ^{2}\left[s^{\operatorname{lag}} \sqrt{\kappa^{\operatorname{lag} / 2}}\right] \\
& z\left(s^{\operatorname{lag}}\right)=s^{\operatorname{lag}}+\epsilon j\left(s^{\operatorname{lag}}\right)^{3} / 6 \\
& z\left(s^{\operatorname{lag}}\right)=\tanh \left[s^{\operatorname{lag}} \sqrt{-\kappa^{\operatorname{lag}} / 2}\right]-\frac{\epsilon j s^{\operatorname{lag}}}{2 \sqrt{-2 \kappa^{\operatorname{lag}}}} \operatorname{sech}^{2}\left[s^{\operatorname{lag}} \sqrt{-\kappa^{\operatorname{lag}} / 2}\right] .
\end{aligned}
$$


It will also be helpful to have these curves expressed using the graph parameter:

$$
\begin{aligned}
& z=x+\frac{\epsilon j}{2 \kappa^{\operatorname{lag}}}\left(1+x^{2}\right) \tan ^{-1} x \\
& z=x+\frac{\epsilon j}{6} x^{3} \\
& z=x+\frac{\epsilon j}{2 \kappa^{\lg }}\left(1-x^{2}\right) \tanh ^{-1} x
\end{aligned}
$$

Finally, we point out that there is a one-parameter family of Laguerre transformations that maps each of these curves onto itself. For example, in the case of zero Laguerre curvature, let

$$
\mu_{c}(z)=\frac{z+\left(c+\frac{\epsilon j c^{3}}{6}\right)}{\left(-\frac{\epsilon j c}{2}\right) z+1}
$$

for $c \in \mathbb{R}$. Then, for $z\left(s^{\operatorname{lag}}\right)=s^{\operatorname{lag}}+\epsilon j\left(s^{\operatorname{lag}}\right)^{3} / 6$, one finds that $\mu_{c} \circ z\left(s^{\operatorname{lag}}\right)=z\left(s^{\operatorname{lag}}+c\right)$.

5. Extremal theory. Liebmann was the first to prove that the curves that are stationary with respect to Laguerre arc length are the curves with constant Laguerre curvature [10].

Later, Maeda proved a similar result for curves of finite length. In particular, he considered competing curves $\widetilde{z}$, relative to Laguerre arc length, for which

$$
\widetilde{z}=z, \quad \widetilde{z}^{\prime}=\lambda z^{\prime}, \quad \frac{\widetilde{z}^{\prime \prime}}{\widetilde{z}^{\prime}}=\lambda \frac{z^{\prime \prime}}{z^{\prime}}+\nu
$$

at the endpoints with $\lambda, \nu$ real. (These conditions preserve the second order information at the endpoints.) Using a variational argument, he showed that curves with stationary Laguerre arc length are arcs of curves with constant Laguerre curvature [13, p.577-581].

In this section, we extend Maeda's result and prove that the stationary curves are maximizers of Laguerre arc length. Our main results are the following.

THEOREM 1. Consider curves (with endpoints) of class $C^{3}$ in $\widehat{\mathbb{D}}$ that can be locally represented as a graph $z=x+j y$ with $d^{3} y / d x^{3} \neq 0$. Among the curves that connect the same fixed osculating endpoint parabolas and belong to the same isotopy class, there is exactly one extremal arc. This extremal arc uniquely maximizes the Laguerre arc length among these curves.

TheOREM 2. Consider curves (with endpoints) of class $C^{3}$ in $\widehat{\mathbb{D}}$ that can be locally represented as a graph $z=x+j y$ with $d^{3} y / d x^{3} \neq 0$. Their Laguerre class is a quadruple $\left(\epsilon, n, \theta_{\mathrm{lag}}, \delta_{\mathrm{lag}}\right)$ where $\epsilon=\operatorname{sgn}\left(d^{3} y / d x^{3}\right)$ and $n$ identifies the isotopy. In each such class, there is exactly one extremal arc up to (direct) Laguerre transformation and this extremal arc uniquely maximizes the Laguerre arc length.

The proof of these results is accomplished in three steps.

First, we use Cauchy-Schwarz estimates to show that the extremal arcs have the greatest Laguerre arc length among competing curves for which (10) is satisfied. We then show that there are enough extremal arcs. In particular, we show that each extremal arc can be assigned a unique quadruple $\left(\epsilon, n, \theta_{\mathrm{lag}}, \delta_{\mathrm{lag}}\right)$ that includes the $\theta_{\mathrm{lag}}$ 
and $\delta_{\text {lag }}$ distances between osculating pointed parabolas at the endpoints and an index $n$ that identifies the isotopy class. Finally, we show that each quadruple identifies a class of curves, with each class containing exactly one extremal arc up to Laguerre transformation.

5.1. Extremals as maximizers of Laguerre arc length. The first step is to show that the extremal arcs, as taken from (7), (8), and (9), are uniquely the maximizers of Laguerre arc length, among competing curves for which (10) is satisfied.

To do this, we consider competing curves that have the form

$$
\begin{aligned}
& z=x+\frac{\epsilon j}{2 \kappa^{\operatorname{lag}}} y(x) \\
& z=x+\frac{\epsilon j}{6} y(x) \\
& z=x+\frac{\epsilon j}{2 \kappa^{\operatorname{lag}}} y(x)
\end{aligned}
$$

for $0 \leq x \leq b$. (Using the one parameter family of Laguerre transformations mentioned at the end of $\S 4$, we may assume the curves originate at the origin.) These curves correspond with the cases $\kappa^{\mathrm{lag}}>0, \kappa^{\mathrm{lag}}=0$, and $\kappa^{\mathrm{lag}}<0$. The restrictions on $y$ and its first two derivatives at the endpoints are expressed in (10). We treat the three cases individually.

(i) An application of Cauchy-Schwarz in the first case gives

$$
\int_{0}^{b}\left(\frac{1}{2 \kappa^{\operatorname{lag}}} \frac{d^{3} y}{d x^{3}}\right)^{1 / 2} d x \leq\left(\int_{0}^{b} \frac{1}{2 \kappa^{\operatorname{lag}}} \frac{d^{3} y}{d x^{3}}\left(1+x^{2}\right) d x\right)^{1 / 2}\left(\int_{0}^{b} \frac{1}{1+x^{2}} d x\right)^{1 / 2}
$$

with equality if and only if there is a constant $c$ for which

$$
\frac{d^{3} y}{d x^{3}}=\frac{c}{\left(1+x^{2}\right)^{2}}
$$

Solving this equation, while requiring that $y$ and its first two derivatives agree with those of $\left(1+x^{2}\right) \tan ^{-1} x$ at $x=0$ and $x=b$ gives $y=\left(1+x^{2}\right) \tan ^{-1} x$.

Moreover, in this case $c=4$, and the inequality simplifies to

$$
\begin{aligned}
\int_{0}^{b}\left(\frac{1}{2 \kappa^{\operatorname{lag}}} \frac{d^{3} y}{d x^{3}}\right)^{1 / 2} d x & \leq\left(\int_{0}^{b} \frac{1}{2 \kappa^{\operatorname{lag}}} \frac{4}{1+x^{2}} d x\right)^{1 / 2}\left(\int_{0}^{b} \frac{1}{1+x^{2}} d x\right)^{1 / 2} \\
& =\sqrt{\frac{2}{\kappa^{\operatorname{lag}}} \tan ^{-1} b=s^{\operatorname{lag}}}
\end{aligned}
$$

exactly the length of the respective extremal arc.

We mention that, as written, the argument applies only to curves that can be written globally as $y=y(x)$ and therefore don't cross $L_{\infty}$. To handle the curves that do cross $L_{\infty}$ (i.e., $n>1$ in what follows) one can substitute $x=\tan \left(s \sqrt{\kappa^{\text {lag }} / 2}\right)$ and perform the same estimates on integrals expressed in terms of $s$. We omit the details. As illustrated later in Figure 8, the situation $n>1$ only applies to the $\kappa^{\text {lag }}>0$ case. The argument has been kept as written both because of its simplicity and because of its similarity to the remaining two cases. 
(ii) An application of Cauchy-Schwarz in the second case gives

$$
\int_{0}^{b}\left(\frac{1}{6} \frac{d^{3} y}{d x^{3}}\right)^{1 / 2} d x \leq\left(\int_{0}^{b} \frac{1}{6} \frac{d^{3} y}{d x^{3}} d x\right)^{1 / 2}\left(\int_{0}^{b} d x\right)^{1 / 2}
$$

with equality if and only if there is a constant $c$ for which

$$
\frac{d^{3} y}{d x^{3}}=c .
$$

Solving this equation, while requiring that $y$ and its first two derivatives agree with those of $x^{3}$ at $x=0$ and $x=b$ gives $y=x^{3}$.

Moreover, in this case $c=6$, and the inequality simplifies to

$$
\int_{0}^{b}\left(\frac{1}{6} \frac{d^{3} y}{d x^{3}}\right)^{1 / 2} d x \leq\left(\int_{0}^{b} \frac{1}{6} \cdot 6 d x\right)^{1 / 2}\left(\int_{0}^{b} d x\right)^{1 / 2}=b=s^{\operatorname{lag}}
$$

exactly the length of the respective extremal arc.

(iii) An application of Cauchy-Schwarz in the third case gives

$$
\int_{0}^{b}\left(\frac{1}{2 \kappa^{\operatorname{lag}}} \frac{d^{3} y}{d x^{3}}\right)^{1 / 2} d x \leq\left(\int_{0}^{b} \frac{1}{2 \kappa^{\operatorname{lag}}} \frac{d^{3} y}{d x^{3}}\left(1-x^{2}\right) d x\right)^{1 / 2}\left(\int_{0}^{b} \frac{1}{1-x^{2}} d x\right)^{1 / 2}
$$

with equality if and only if there is a constant $c$ for which

$$
\frac{d^{3} y}{d x^{3}}=\frac{c}{\left(1-x^{2}\right)^{2}} .
$$

Solving this equation, while requiring that $y$ and its first two derivatives agree with those of $\left(1-x^{2}\right) \tanh ^{-1} x$ at $x=0$ and $x=b$ gives $y=\left(1-x^{2}\right) \tanh ^{-1} x$.

Moreover, in this case $c=-4$, and the inequality simplifies to

$$
\begin{aligned}
\int_{0}^{b}\left(\frac{1}{2 \kappa^{\operatorname{lag}}} \frac{d^{3} y}{d x^{3}}\right)^{1 / 2} d x & \leq\left(\int_{0}^{b}-\frac{1}{2 \kappa^{\operatorname{lag}}} \frac{4}{1-x^{2}} d x\right)^{1 / 2}\left(\int_{0}^{b} \frac{1}{1-x^{2}} d x\right)^{1 / 2} \\
& =\sqrt{-\frac{2}{\kappa^{\lg }}} \tanh ^{-1} b=s^{\operatorname{lag}}
\end{aligned}
$$

exactly the length of the respective extremal arc.

5.2. Values of distance functions along the extremals. The next step is to show there are enough extremals in the sense that, up to direct Laguerre transformation, there is exactly one extremal arc that corresponds with a given quadruple $\left(\epsilon, n, \theta_{\mathrm{lag}}, \delta_{\mathrm{lag}}\right)$. The role played by the signature is obvious, so it is only necessary to demonstrate how a given triple $\left(n, \theta_{\mathrm{lag}}, \delta_{\mathrm{lag}}\right)$ determines a pair $\left(s^{\mathrm{lag}}, \kappa^{\mathrm{lag}}\right)$.

Coxeter distance. For a curve $z(t)=x(t)+j y(t)$ we use a Taylor expansion to find the osculating parabola at $z(a)$. It has the form

$$
\mathbf{p}_{a}: y=y(a)+\frac{d y}{d x}(x-x(a))+\frac{1}{2} \frac{d^{2} y}{d x^{2}}(x-x(a))^{2}
$$


where the derivatives are evaluated at $t=a$. We find

$$
\frac{d y}{d x}=\frac{d y}{d t} \frac{d t}{d x}=\frac{y^{\prime}(t)}{x^{\prime}(t)}
$$

and

$$
\frac{d^{2} y}{d x^{2}}=\frac{d}{d t}\left(\frac{d y}{d t} \frac{d t}{d x}\right) \frac{d t}{d x}=\frac{y^{\prime \prime}(t) x^{\prime}(t)-y^{\prime}(t) x^{\prime \prime}(t)}{x^{\prime}(t)^{3}} .
$$

So the osculating parabola is

$$
y=y(a)+\frac{y^{\prime}(a)}{x^{\prime}(a)}(x-x(a))+\frac{1}{2} \frac{y^{\prime \prime}(a) x^{\prime}(a)-y^{\prime}(a) x^{\prime \prime}(a)}{x^{\prime}(a)^{3}}(x-x(a))^{2} .
$$

Now for an extremal $z$ connecting $z(0)$ to $z\left(s^{\mathrm{lag}}\right)$, we compute the distance between the osculating parabolas $\mathbf{p}_{0}$ and $\mathbf{p}_{s^{\text {lag }}}$. As before, $\kappa^{\text {lag }}$ is the Laguerre curvature of the extremal and $s^{\text {lag }}$ is the Laguerre length. Then following some routine computations,

$$
\begin{aligned}
\kappa^{\operatorname{lag}}>0: \delta_{\operatorname{lag}} & =\left(-1+\kappa^{\operatorname{lag}}\left(s^{\operatorname{lag}}\right)^{2}+\cos \left[s^{\operatorname{lag} \sqrt{2 \kappa^{\operatorname{lag}}}}\right]\right)^{1 / 2} /\left(\sqrt{2} \kappa^{\operatorname{lag}}\right) \\
\kappa^{\operatorname{lag}}=0: & \delta_{\operatorname{lag}}=\left(s^{\operatorname{lag}}\right)^{2} / \sqrt{12} \\
\kappa^{\operatorname{lag}}<0: & \delta_{\operatorname{lag}}=\left(-1+\kappa^{\operatorname{lag}}\left(s^{\operatorname{lag}}\right)^{2}+\cosh \left[s^{\operatorname{lag}} \sqrt{-2 \kappa^{\operatorname{lag}}}\right]\right)^{1 / 2} /\left(-\sqrt{2} \kappa^{\operatorname{lag}}\right) .
\end{aligned}
$$

Since $\cosh i x=\cos x$, the three expressions are interconnected-the case $\kappa^{\text {lag }}=0$ can be taken as the limiting case $\kappa^{\operatorname{lag}} \rightarrow 0^{ \pm}$.

Kerzman-Stein distance. For the curve $z(t)=x(t)+j y(t)$ we also determine the parabola that is tangent to $z$ at $z(a)$ and passes through $z(b)$. It has the form

$$
\mathbf{p}_{a b}: y=y(a)+\frac{y^{\prime}(a)}{x^{\prime}(a)}(x-x(a))+\varphi(a, b) \cdot(x-x(a))^{2},
$$

where the condition $y=y(b)$ when $x=x(b)$ requires

$$
\varphi(a, b)=\frac{y(b)-y(a)}{(x(b)-x(a))^{2}}-\frac{y^{\prime}(a)}{x^{\prime}(a)} \frac{1}{x(b)-x(a)} .
$$

For an extremal $z$ connecting $z(0)$ to $z\left(s^{\mathrm{lag}}\right)$, we compute the distance between the parabolas $\mathbf{p}_{0 s^{\operatorname{lag}}}$ and $\mathbf{p}_{s^{\operatorname{lag} 0}}$ constructed from the supporting line elements at $z(0)$ and $z\left(s^{\mathrm{lag}}\right)$. As before, $\kappa^{\text {lag }}$ is the Laguerre curvature of the extremal and $s^{\text {lag }}$ is the Laguerre length. Again following some routine computations,

$$
\begin{aligned}
& \kappa^{\operatorname{lag}}>0: \theta_{\operatorname{lag}}=\left|1-s^{\operatorname{lag}} \sqrt{\kappa^{\operatorname{lag} / 2}} \cot \left[s^{\operatorname{lag}} \sqrt{\kappa^{\operatorname{lag}} / 2}\right]\right| / \kappa^{\operatorname{lag}} \\
& \kappa^{\operatorname{lag}}=0: \theta_{\operatorname{lag}}=\left(s^{\operatorname{lag}}\right)^{2} / 6 \\
& \kappa^{\operatorname{lag}}<0: \theta_{\operatorname{lag}}=\left|1-s^{\operatorname{lag}} \sqrt{-\kappa^{\operatorname{lag} / 2}} \operatorname{coth}\left[s^{\operatorname{lag}} \sqrt{-\kappa^{\operatorname{lag}} / 2}\right]\right| /\left(-\kappa^{\operatorname{lag}}\right) .
\end{aligned}
$$

As before, the expressions are interconnected - the case $\kappa^{\text {lag }}=0$ can be taken as the limiting case $\kappa^{\text {lag }} \rightarrow 0^{ \pm}$.

Recovering $\left(s^{\mathrm{lag}}, \kappa^{\mathrm{lag}}\right)$ from a given triple $\left(n, \theta_{\mathrm{lag}}, \delta_{\mathrm{lag}}\right)$. We now specify the index $n \in \mathbb{N}$ that identifies the isotopy class and we describe a bijection $\mathbb{R}^{+} \times \mathbb{R} \leftrightarrow$ 
$\mathbb{N} \times \widehat{\mathbb{R}}^{+} \times \mathbb{R}^{+}$by which $\left(s^{\text {lag }}, \kappa^{\text {lag }}\right) \leftrightarrow\left(n, \theta_{\text {lag }}, \delta_{\text {lag }}\right)$. If $n$ is odd we take $\widehat{\mathbb{R}}^{+}=(0, \infty]$ and if $n$ is even we take $\widehat{\mathbb{R}}^{+}=[0, \infty)$. Geometrically, the index is one greater than roughly twice the number of times the curve crosses the line at infinity.

Consider first the case $\kappa^{\mathrm{lag}}<0$ and notice that

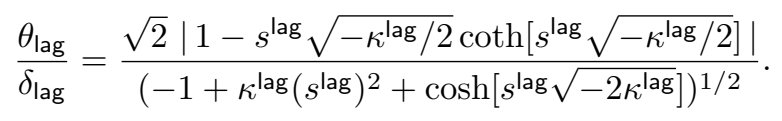

After introducing an intermediate variable $t=s^{\operatorname{lag}} \sqrt{-2 \kappa^{\operatorname{lag}}}$, one finds that the ratio $\theta_{\mathrm{lag}} / \delta_{\mathrm{lag}}$ has the same range as the decreasing function

$$
f(t)=|2-t \operatorname{coth}(t / 2)| /\left(-2-t^{2}+2 \cosh t\right)^{1 / 2} .
$$

As can be seen in Figure 5 , this range is $(0,1 / \sqrt{3})$. So in case $\kappa^{\operatorname{lag}}<0$, we set $n=1$

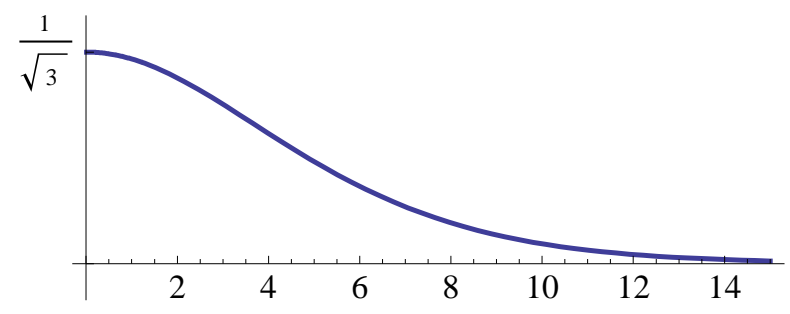

FIG. 5. Plot of $f(t)=|2-t \operatorname{coth}(t / 2)| /\left(-2-t^{2}+2 \cosh t\right)^{1 / 2}$.

and find that $\left(s^{\operatorname{lag}}, \kappa^{\mathrm{lag}}\right)$ corresponds with a triple $\left(1, \theta_{\mathrm{lag}}, \delta_{\mathrm{lag}}\right)$ for $\theta_{\mathrm{lag}}<\delta_{\mathrm{lag}} / \sqrt{3}$.

Conversely, a triple $\left(1, \theta_{\mathrm{lag}}, \delta_{\mathrm{lag}}\right)$ with $\theta_{\mathrm{lag}}<\delta_{\mathrm{lag}} / \sqrt{3}$ determines a pair $\left(s^{\mathrm{lag}}, \kappa^{\mathrm{lag}}\right)$ with $\kappa^{\text {lag }}<0$. To see this, first find $t=s^{\operatorname{lag}} \sqrt{-2 \kappa^{\operatorname{lag}}}$ by solving $t=f^{-1}\left(\theta_{\text {lag }} / \delta_{\text {lag }}\right)$. Then set $\kappa^{\operatorname{lag}}=\left(-2-t^{2}+2 \cosh t\right)^{1 / 2} /\left(-2 \delta_{\operatorname{lag}}\right)$ and $s^{\operatorname{lag}}=t / \sqrt{-2 \kappa^{\operatorname{lag}}}$.

The case $\kappa^{\operatorname{lag}}=0$ corresponds with $n=1$ and $\theta_{\operatorname{lag}}=\delta_{\operatorname{lag}} / \sqrt{3}=s^{\operatorname{lag}^{2}} / 6$. So for a triple $\left(1, \theta_{\text {lag }}, \delta_{\text {lag }}\right)$ with $\theta_{\text {lag }}=\delta_{\text {lag }} / \sqrt{3}$, one recovers $\kappa^{\text {lag }}=0$ and $s^{\text {lag }}=\left(6 \theta_{\text {lag }}\right)^{1 / 2}=$ $\left(\sqrt{12} \delta_{\text {lag }}\right)^{1 / 2}$.

An analysis similar to the case for $\kappa^{\text {lag }}<0$ works also for $\kappa^{\text {lag }}>0$. Notice that

$$
\frac{\theta_{\operatorname{lag}}}{\delta_{\operatorname{lag}}}=\frac{\sqrt{2}\left|1-s^{\operatorname{lag}} \sqrt{\kappa^{\operatorname{lag} / 2}} \cot \left[s^{\operatorname{lag}} \sqrt{\kappa^{\operatorname{lag} / 2}}\right]\right|}{\left(-1+\kappa^{\operatorname{lag}}\left(s^{\operatorname{lag}}\right)^{2}+\cos \left[s^{\operatorname{lag}} \sqrt{2 \kappa^{\operatorname{lag}}}\right]\right)^{1 / 2}} .
$$

Like before we substitute $t=s^{\operatorname{lag}} \sqrt{2 \kappa^{\operatorname{lag}}}$. The ratio $\theta_{\mathrm{lag}} / \delta_{\mathrm{lag}}$ now has the same range as the increasing function

$$
g(t)=|2-t \cot (t / 2)| /\left(-2+t^{2}+2 \cos t\right)^{1 / 2} .
$$

For values $0<t \leq 2 \pi$, this range is $(1 / \sqrt{3}, \infty]$. Then for $\left(s^{\operatorname{lag}}, \kappa^{\operatorname{lag}}\right)$ with $\kappa^{\operatorname{lag}}>0$ and

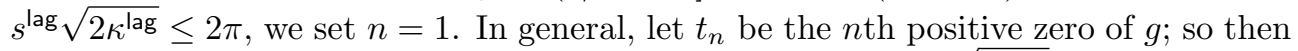
$t_{n} \approx(2 n+1) \pi$ for large $n$. For $\left(s^{\operatorname{lag}}, \kappa^{\operatorname{lag}}\right)$ with $\kappa^{\operatorname{lag}}>0$ and $s^{\operatorname{lag}} \sqrt{2 \kappa^{\operatorname{lag}}}>2 \pi$, we define $n$ according to the rule

i) if $2 \pi j<s^{\operatorname{lag} \sqrt{2 \kappa^{\operatorname{lag}}}} \leq t_{j}$, then $n=2 j$

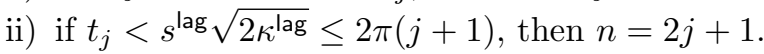




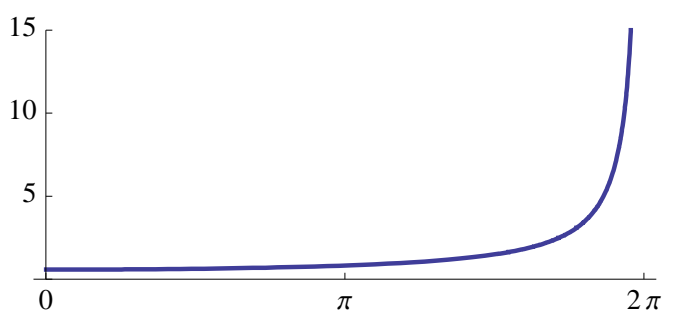

FIG. 6. Plot of $g(t)=|2-t \cot (t / 2)| /\left(-2+t^{2}+2 \cos t\right)^{1 / 2}$.

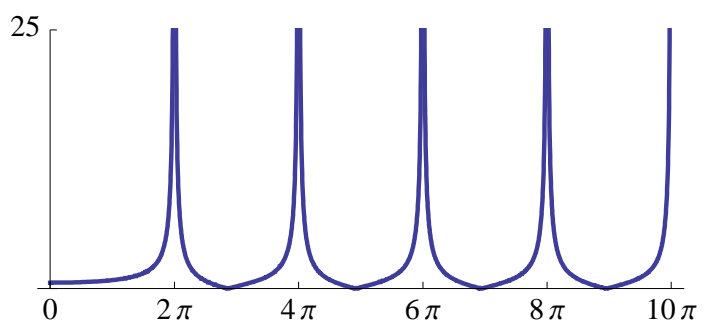

FIG. 7. Plot of $g(t)=|2-t \cot (t / 2)| /\left(-2+t^{2}+2 \cos t\right)^{1 / 2}$.

Here is how to recover the pair $\left(s^{\mathrm{lag}}, \kappa^{\mathrm{lag}}\right)$ with $\kappa^{\mathrm{lag}}>0$ for a triple $\left(n, \theta_{\text {lag }}, \delta_{\text {lag }}\right)$ with $\theta_{\text {lag }}>\delta_{\text {lag }} / \sqrt{3}$ in case $n=1$, and with arbitrary $\theta_{\text {lag }}, \delta_{\text {lag }}$ in case $n>1$. If

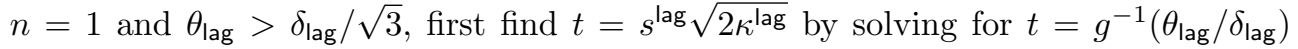
with $0<t \leq 2 \pi$. (In particular, if $\theta_{\text {lag }}=\infty$ then $t=2 \pi$.) If $n>1$, then solve for $t=g^{-1}\left(\theta_{\text {lag }} / \delta_{\text {lag }}\right)$ with

i) $2 \pi j<t \leq t_{j}$, if $n=2 j$

ii) $t_{j}<t \leq 2 \pi(j+1)$, if $n=2 j+1$.

Finally, set $\kappa^{\text {lag }}=\left(-2+t^{2}+2 \cos t\right)^{1 / 2} /\left(2 \delta_{\text {lag }}\right)$ and $s^{\text {lag }}=t / \sqrt{2 \kappa^{\operatorname{lag}}}$.

Figure 8 helps illustrate the correspondence $\left(s^{\mathrm{lag}}, \kappa^{\mathrm{lag}}\right) \leftrightarrow\left(n, \theta_{\mathrm{lag}}, \delta_{\mathrm{lag}}\right)$.
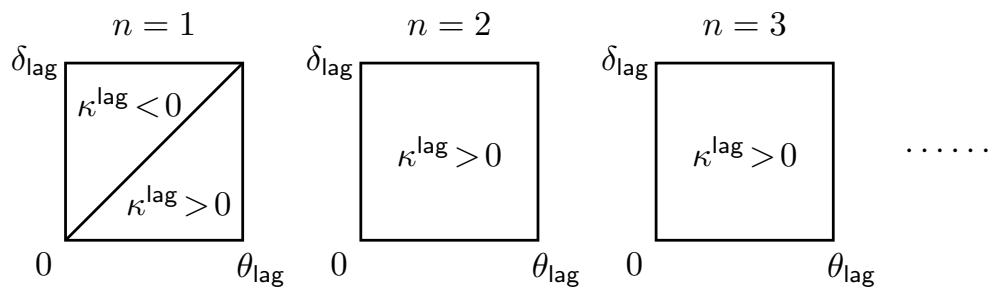

FIG. 8. The correspondence $\left(s^{\mathrm{lag}}, \kappa^{\mathrm{lag}}\right) \leftrightarrow\left(n, \theta_{\mathrm{lag}}, \delta_{\mathrm{lag}}\right)$ for the Laguerre invariants among arcs of extremal curves.

We reiterate that a quadruple $\left(\epsilon, n, \theta_{\text {lag }}, \delta_{\text {lag }}\right)$ now identifies an equivalence class of curves that contains exactly one extremal arc up to direct Laguerre transformation.

5.3. Normalization of the endpoint parabolas. The final step is to show that a curve can be normalized using only its signature and the $\theta_{\text {lag }}$ and $\delta_{\text {lag }}$ distances between the osculating parabolas at the endpoints. Following this normalization, it qualifies as one of the competing curves in the sense of $\S 5.1$. 
Normalization. We show first how to place a curve in normal position with respect to the osculating parabolas at the endpoints. In particular, we normalize the curve so that the initial pointed parabola is $y=-x^{2}$ and is pointed at 0 , and the terminal pointed parabola is pointed at 1 .

To do this, first use a map $z \rightarrow z+\left(x_{0}+j y_{0}\right)$ to transform the initial parabola into a parabola that is pointed at 0 . Follow this with a map $z \rightarrow\left(1+j y_{0}\right) z$ that makes the tangent line horizontal at 0 . The direct Laguerre transformations that preserve this configuration have the form $\pm d^{-1} z /(c z+d)$ for $c \in \mathbb{D}$ and $0 \neq d \in \mathbb{R}$. Replacing $d$ with $-d$ results in the same kind of transformation, so one may assume that $d>0$. By choosing $c$ appropriately, one can then make the terminal parabola to be pointed at 1. The remaining Laguerre transformations then have the form $d^{-1} z /((1 / d-d) z+d)$ or $d^{-1} z /((1 / d+d) z-d)$ for $0<d<\infty$. These maps now adjust the concavity at the origin by a factor of $d^{2}$ or $-d^{2}$, respectively. So with a suitable choice for $d$, one may assume that the initial parabola is $y=-x^{2}$.

Dependence on $\left(\theta_{\mathrm{lag}}, \delta_{\mathrm{lag}}\right)$. Wंe now show how the signature $\epsilon$ and the pair $\left(\theta_{\mathrm{lag}}, \delta_{\mathrm{lag}}\right)$ with $\theta_{\mathrm{lag}}, \delta_{\mathrm{lag}}>0$ uniquely determine the terminal parabola.

In normal position, the initial parabola $\mathbf{p}_{0}^{*}$ has equation $y=-x^{2}$, and the terminal parabola $\mathbf{p}_{1}^{*}$ has equation $y=a x^{2}+(b-2 a) x+(-b+a)$ for $a, b \in \mathbb{R}$. (There are restrictions on $a, b$ to prevent the parabolas from intersecting; specifically, $b^{2}+4 b<$ 4a.) The constructed parabolas $\mathbf{p}_{01}$ and $\mathbf{p}_{10}$ are then $y=0$ and $y=b x^{2}-b x$.

We find

$$
\theta_{\operatorname{lag}}\left(\mathbf{p}_{0}^{*}, \mathbf{p}_{1}^{*}\right)=\sqrt{\Delta\left(\mathbf{p}_{01}, \mathbf{p}_{10}\right)}=\sqrt{(-b)^{2}-4(b)(0)}=|b|
$$

and

$$
\begin{aligned}
\delta_{\operatorname{lag}}\left(\mathbf{p}_{0}^{*}, \mathbf{p}_{1}^{*}\right) & =\sqrt{-\Delta\left(\mathbf{p}_{0}, \mathbf{p}_{1}\right)} \\
& =\sqrt{-(b-2 a)^{2}+4(a+1)(-b+a)} \\
& =\sqrt{-b^{2}-4 b+4 a} .
\end{aligned}
$$

Solving these equations gives $b= \pm \theta_{\operatorname{lag}}$ and $a=\delta_{\operatorname{lag}}^{2} / 4+b+b^{2} / 4$. So there are two possibilities for the pair $(a, b)$ :

i) $(a, b)=\left(\delta_{\operatorname{lag}}^{2} / 4+\theta_{\operatorname{lag}}+\theta_{\operatorname{lag}}^{2} / 4,+\theta_{\operatorname{lag}}\right)$

ii) $(a, b)=\left(\delta_{\mathrm{lag}} / 4-\theta_{\mathrm{lag}}+\theta_{\mathrm{lag}}^{2} / 4,-\theta_{\mathrm{lag}}\right)$

Figure 9 illustrates the two cases when $\theta_{\text {lag }}=1$ and $\delta_{\text {lag }}=2$. Notice that the slope of
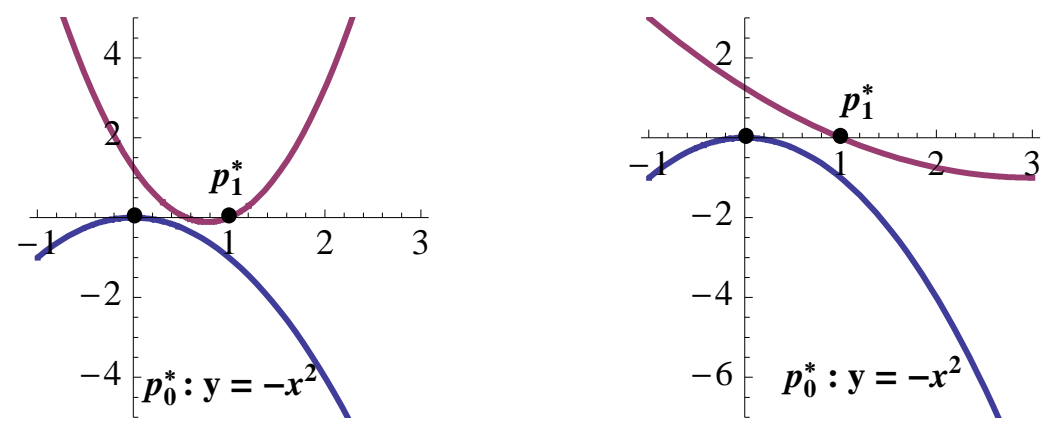

FIG. 9. Cases (i) and (ii): configurations for the endpoint parabolas after a curve has been normalized $\left(\theta_{\mathrm{lag}}=1, \delta_{\mathrm{lag}}=2\right)$. 
$\mathbf{p}_{1}^{*}$ at 1 is $b= \pm \theta^{2} / 6$. We mention that one can verify directly that an extremal arc with signature $\epsilon=+1$ or -1 corresponds, respectively, with case (i) or (ii). The same then must be true for general curves, too, else it would be possible to construct a homotopy between curves of opposite signature (from a general curve to an extremal arc) so that all curves in the homotopy have the same configuration of case (i) or (ii). This leads to a contradiction, as for some curve along the homotopy one has $\int\left(d^{3} y / d x^{3}\right) d x=0$, which cannot possibly fit either configuration.

So $\epsilon=+1$ or -1 then corresponds with case (i) or (ii), respectively, and so it is possible to normalize the second order information at the endpoints using only the signature and the $\theta_{\operatorname{lag}}$ and $\delta_{\text {lag }}$ distances between the parabolas at the endpoints.

Completion of the proof of Theorems 1 and 2. Consider a curve $\gamma \in \widehat{\mathbb{D}}$ of fixed signature whose endpoints are finite. (It may be necessary to use that a Laguerre transformation to accomplish this.) One computes the $\theta_{\text {lag }}$ and $\delta_{\text {lag }}$ distances between the osculating parabolas at the endpoints, and the index $n$ by considering the number of times the curve crosses $L_{\infty}$. Following $\S 5.2$ and $\S 5.3$, there is exactly one extremal arc that has the same Laguerre class $\left(\epsilon, n, \theta_{\mathrm{lag}}, \delta_{\mathrm{lag}}\right)$ and which has the same osculating parabolas at the endpoints. Following $\S 5.1$, the extremal arc has the greatest Laguerre arc length among all curves in this class.

6. Cauchy integrals and extremal curves. We here provide an explicit description of the Cauchy transform for a curve $\gamma$ of constant negative Laguerre curvature. The transform is defined by

$$
\mathcal{C} f(z) \stackrel{\text { def }}{=} \sqrt{\frac{2}{\pi}} P . V \cdot \int_{\gamma} \frac{f(w) d w}{w-z}
$$

where $f \in L^{2}(\gamma)$ and $z \in \gamma$. For the case of Möbius invariant geometry over $\mathbb{C}$, a description of the Cauchy transform for logarithmic spirals is given in [4]. (Logarithmic spirals and their Möbius images are the curves of constant Möbius curvature.)

Following [1], it is natural to express the transform using half-order differentials. In this setting, one can verify directly that the Cauchy kernel,

$$
\sqrt{\frac{2}{\pi}} \frac{\sqrt{d w} \sqrt{d z}}{w-z}
$$

is invariant under direct Laguerre transformations. (For the moment, the multiplicative constant is arbitrary.) The Cauchy transform then acts by integrating expressions of the form $f(w) \sqrt{d w}$ against the kernel. By analyzing the kernel, one can obtain a complete description of the Cauchy transform.

This is done best in terms of the Laguerre arc length coordinate. For simpler notation, we replace $\kappa=\kappa^{\mathrm{lag}}$ and $s=s^{\mathrm{lag}}$, then using the Laguerre parameter, we set $w=z(s)$ and $z=z(t)$. A routine computation shows that

$$
z^{\prime}(s)=\left(\frac{-\kappa}{2}\right)^{1 / 2} \operatorname{sech}^{2}\left(s \sqrt{\frac{-\kappa}{2}}\right)\left[1+\frac{\epsilon j}{2 \kappa}\left(1-s \sqrt{-2 \kappa} \tanh \left(s \sqrt{\frac{-\kappa}{2}}\right)\right)\right],
$$

and therefore,

$$
\sqrt{z^{\prime}(s)}=\left(\frac{-\kappa}{2}\right)^{1 / 4} \operatorname{sech}\left(s \sqrt{\frac{-\kappa}{2}}\right)\left[1+\frac{\epsilon j}{4 \kappa}\left(1-s \sqrt{-2 \kappa} \tanh \left(s \sqrt{\frac{-\kappa}{2}}\right)\right)\right]
$$


with similar expressions for $z^{\prime}(t)$ and $\sqrt{z^{\prime}(t)}$.

In terms of the Laguerre parameter, the Cauchy kernel can be expressed as

$$
\sqrt{\frac{2}{\pi}} \frac{\sqrt{d w} \sqrt{d z}}{w-z}=\sqrt{\frac{2}{\pi}} \frac{\sqrt{z^{\prime}(s)} \sqrt{z^{\prime}(t)}}{z(s)-z(t)} \sqrt{d s} \sqrt{d t}
$$

where after routine computation,

$$
\begin{aligned}
\frac{\sqrt{z^{\prime}(s)} \sqrt{z^{\prime}(t)}}{z(s)-z(t)} & =\left(\frac{-\kappa}{2}\right)^{1 / 2} \operatorname{csch}\left((s-t) \sqrt{\frac{-\kappa}{2}}\right) \\
+ & \epsilon j \operatorname{csch}\left((s-t) \sqrt{\frac{-\kappa}{2}}\right)\left[-\frac{1}{2 \sqrt{-2 \kappa}}+\frac{s-t}{4} \operatorname{coth}\left((s-t) \sqrt{\frac{-\kappa}{2}}\right)\right] .
\end{aligned}
$$

Evidently, in terms of the Laguerre parameter, the Cauchy transform is a convolution operator on $\mathbb{R}$, so it can be fully understood through the Fourier transform.

In particular, if we define

$$
\begin{array}{r}
\mathcal{F}(h)(\xi) \stackrel{\text { def }}{=} \frac{i}{\sqrt{2 \pi}} \int_{-\infty}^{+\infty} h(s) e^{-i s \xi} d s \\
\mathcal{F}^{-1}(h)(s) \stackrel{\text { def }}{=} \frac{-i}{\sqrt{2 \pi}} \int_{-\infty}^{+\infty} h(\xi) e^{+i s \xi} d \xi
\end{array}
$$

then after taking Fourier transforms, the Cauchy transform is equivalent to multiplication by the function $\sqrt{2 / \pi} \cdot \mathcal{F}(\Psi)$ where

$$
\Psi(s)=\sqrt{\frac{-\kappa}{2}} \operatorname{csch}\left(s \sqrt{\frac{-\kappa}{2}}\right)+\epsilon j \operatorname{csch}\left(s \sqrt{\frac{-\kappa}{2}}\right)\left[-\frac{1}{2 \sqrt{-2 \kappa}}+\frac{s}{4} \operatorname{coth}\left(s \sqrt{\frac{-\kappa}{2}}\right)\right] .
$$

A routine computation reveals that

$$
\sqrt{\frac{2}{\pi}} \cdot \mathcal{F}(\Psi)(\xi)=\tanh \left(\frac{\pi \xi}{\sqrt{-2 \kappa}}\right)+\frac{\epsilon j \pi \xi}{(-2 \kappa)^{3 / 2}} \operatorname{sech}^{2}\left(\frac{\pi \xi}{\sqrt{-2 \kappa}}\right) .
$$

Surprisingly, then, after replacing the transform parameter $\xi$ using $\eta=-\pi \xi / \kappa$, one finds that the Cauchy transform (on a curve of constant negative Laguerre curvature) acts as multiplication by the function

$$
\overline{z(\eta)}=\tanh \left(\eta \sqrt{\frac{-\kappa}{2}}\right)+\frac{\epsilon j \eta}{2 \sqrt{-2 \kappa}} \operatorname{sech}^{2}\left(\eta \sqrt{\frac{-\kappa}{2}}\right)
$$

exactly the conjugate of the parameterization for the given curve!

7. Distortion. In this section we examine the rate at which the Riemann sums converge to the Laguerre arc length of a curve.

7.1. Laguerre curvature. As in Möbius geometry, one can use the invariance of the Schwarzian to obtain an expression for Laguerre curvature. In particular, the derivative $\{z ; t\}$ is invariant under transformations of the dependent variable, but not under changes of the parameter. So using the formula for the change in Schwarzian (see (3)) one can use a change of parameter to normalize Dual $\{z ; t\} \equiv$ 
$\epsilon=\operatorname{sgn}\left(d^{3} y / d x^{3}\right)$. The normalized parameter is then in fact the Laguerre parameter. That is, Dual $\left\{z ; s^{\operatorname{lag}}\right\} \equiv \epsilon$.

To verify this, suppose $z(t)=x(t)+j y(t)$ parameterizes a curve for some real parameter $t$. Then

$$
\frac{z^{\prime \prime}}{z^{\prime}}=\frac{x^{\prime \prime}+j y^{\prime \prime}}{x^{\prime}+j y^{\prime}}=\frac{x^{\prime \prime}}{x^{\prime}}+j\left(\frac{y^{\prime}}{x^{\prime}}\right)^{\prime}=\frac{x^{\prime \prime}}{x^{\prime}}+j\left(x^{\prime}\right) \frac{d^{2} y}{d x^{2}}
$$

and

$$
\{z ; t\}=\left(\frac{z^{\prime \prime}}{z^{\prime}}\right)^{\prime}-\frac{1}{2}\left(\frac{z^{\prime \prime}}{z^{\prime}}\right)^{2}=\{x ; t\}+j\left(x^{\prime}\right)^{2} \frac{d^{3} y}{d x^{3}},
$$

where primes indicate derivatives with respect to $t$. The condition Dual $\{z ; t\} \equiv \epsilon$ then reduces to

$$
\left(x^{\prime}\right)^{2} \frac{d^{3} y}{d x^{3}} \equiv \epsilon
$$

from which it follows that $\sqrt{\epsilon d^{3} y / d x^{3}} d x=d t$ and, therefore, Dual $\left\{z ; s^{\operatorname{lag}}\right\} \equiv \epsilon$.

Following this normalization, the Laguerre curvature $\kappa^{\text {lag }}$ is defined to be the real part of the Schwarzian. In particular, $\kappa^{\text {lag }} \stackrel{\text { def }}{=}$ Real $\left\{z ; s^{\text {lag }}\right\}$. Putting all this together one has

$$
\left\{z ; s^{\operatorname{lag}}\right\}=\kappa^{\operatorname{lag}}+\epsilon j=\left\{x ; s^{\operatorname{lag}}\right\}+j\left(x^{\prime}\right)^{2} \frac{d^{3} y}{d x^{3}} .
$$

It can be helpful sometimes to have $\kappa^{\text {lag }}$ expressed using a general parameterization [14, p.555]. In particular, a routine computation (from (3)) shows that for a general parameter $t$, with $\{z ; t\}=A+j B$, one has

$$
\kappa^{\operatorname{lag}}=\epsilon\left(8 A B^{2}-4 B B^{\prime \prime}+5\left(B^{\prime}\right)^{2}\right) /\left(8 B^{3}\right) .
$$

7.2. Distortion of $\sqrt[4]{12} \delta_{\operatorname{lag}}^{1 / 2}$. Start with a curve $z=x+j y$ parameterized by Laguerre arc length. The osculating parabola at $z\left(s_{i}^{\operatorname{lag}}\right)$ can be expressed as

$$
\mathbf{p}_{i}: y=y_{i}+y_{i}^{\prime}\left(x-x_{i}\right)+\frac{1}{2} y_{i}^{\prime \prime}\left(x-x_{i}\right)^{2} .
$$

(The primes on $y$ are derivatives with respect to $x$, and subscripts on $x$ and $y$ are evaluations at $s_{i}^{\operatorname{lag}}$, which is the Laguerre arc length parameter at points of a partition.) If $\Delta x_{i}=x_{i+1}-x_{i}$, then after a routine computation, a first expansion gives

$$
\begin{aligned}
& \Delta\left(\mathbf{p}_{i}, \mathbf{p}_{i+1}\right)= \\
& \quad-\frac{\left(y_{i}^{\prime \prime \prime}\right)^{2}}{12}\left(\Delta x_{i}\right)^{4}-\frac{y_{i}^{\prime \prime \prime} y_{i}^{(4)}}{12}\left(\Delta x_{i}\right)^{5}-\frac{5\left(y_{i}^{(4)}\right)^{2}+11 y_{i}^{\prime \prime \prime} y_{i}^{(5)}}{360}\left(\Delta x_{i}\right)^{6}+o\left(\left(\Delta x_{i}\right)^{6}\right) .
\end{aligned}
$$

Since $\delta_{\text {lag }}=\sqrt{-\Delta}$, a further expansion reveals

$$
\begin{aligned}
& \delta_{\operatorname{lag}}\left(\mathbf{p}_{i}, \mathbf{p}_{i+1}\right)= \\
& \quad \epsilon\left[\frac{y_{i}^{\prime \prime \prime}}{2 \sqrt{3}}\left(\Delta x_{i}\right)^{2}+\frac{y_{i}^{(4)}}{4 \sqrt{3}}\left(\Delta x_{i}\right)^{3}+\frac{-5\left(y_{i}^{(4)}\right)^{2}+22 y_{i}^{\prime \prime \prime} y_{i}^{(5)}}{240 \sqrt{3} y_{i}^{\prime \prime \prime}}\left(\Delta x_{i}\right)^{4}\right]+o\left(\left(\Delta x_{i}\right)^{4}\right),
\end{aligned}
$$


and using together (14) and (15),

$$
\sqrt[4]{12} \delta_{\operatorname{lag}}\left(\mathbf{p}_{i}, \mathbf{p}_{i+1}\right)^{1 / 2}=\Delta s_{i}^{\operatorname{lag}}-\frac{\kappa_{i}^{\operatorname{lag}}}{60}\left(\Delta s_{i}^{\operatorname{lag}}\right)^{3}+o\left(\left(\Delta s_{i}^{\operatorname{lag}}\right)^{3}\right) .
$$

Notice that this gives an under-approximation to the Laguerre arc length when $\kappa^{\text {lag }}>$ 0 ; it gives an over-approximation when $\kappa^{\text {lag }}<0$.

7.3. Distortion of $\sqrt{6} \theta_{\operatorname{lag}}{ }^{1 / 2}$. Again, take a curve $z=x+j y$ parameterized by Laguerre arc length. The parabola tangent to $z$ at $z\left(s_{i}^{\operatorname{lag}}\right)$ and through $z\left(s_{i+1}^{\operatorname{lag}}\right)$ can be expressed as

$$
\mathbf{p}_{i, i+1}: y=y_{i}+y_{i}^{\prime}\left(x-x_{i}\right)+\frac{y_{i+1}-y_{i}-y_{i}^{\prime}\left(\Delta x_{i}\right)}{\left(\Delta x_{i}\right)^{2}}\left(x-x_{i}\right)^{2} .
$$

Similarly, the parabola tangent to $z$ at $z\left(s_{i+1}^{\operatorname{lag}}\right)$ and through $z\left(s_{i}^{\operatorname{lag}}\right)$ can be expressed as

$$
\mathbf{p}_{i+1, i}: y_{i+1}+y_{i+1}^{\prime}\left(x-x_{i+1}\right)+\frac{y_{i}-y_{i+1}+y_{i+1}^{\prime}\left(\Delta x_{i}\right)}{\left(\Delta x_{i}\right)^{2}}\left(x-x_{i+1}\right)^{2} .
$$

Since the parabolas are known to intersect at $z\left(s_{i}^{\operatorname{lag}}\right)$, it is simpler to compute the angle of intersection using the difference in slope of these two parabolas at $x\left(s_{i}^{\operatorname{lag}}\right)$. A first expansion gives

$$
\begin{aligned}
\left(\Delta\left(\mathbf{p}_{i, i+1}, \mathbf{p}_{i+1, i}\right)\right)^{1 / 2} & =\epsilon\left[y_{i}^{\prime}+y_{i+1}^{\prime}-2 \frac{y_{i+1}-y_{i}}{\Delta x_{i}}\right] \\
& =\epsilon\left[\frac{y_{i}^{\prime \prime \prime}}{6}\left(\Delta x_{i}\right)^{2}+\frac{y_{i}^{(4)}}{12}\left(\Delta x_{i}\right)^{3}+\frac{y_{i}^{(5)}}{40}\left(\Delta x_{i}\right)^{4}\right]+o\left(\left(\Delta x_{i}\right)^{4}\right) .
\end{aligned}
$$

Since $\theta_{\text {lag }}=\sqrt{\Delta}$, another expansion using also (14) and (15) gives

$$
\sqrt{6} \theta_{\operatorname{lag}}\left(\mathbf{p}_{i}, \mathbf{p}_{i+1}\right)^{1 / 2}=\Delta s_{i}^{\operatorname{lag}}+\frac{\kappa_{i}^{\operatorname{lag}}}{60}\left(\Delta s_{i}^{\operatorname{lag}}\right)^{3}+o\left(\left(\Delta s_{i}^{\operatorname{lag}}\right)^{3}\right) .
$$

Notice that this gives an over-approximation to the Laguerre arc length when $\kappa^{\text {lag }}>0$; it gives an under-approximation when $\kappa^{\text {lag }}<0$.

7.4. Triangle and reverse triangle inequality. We point out that as a consequence of these estimates, the quantity $\sqrt[4]{12} \delta_{\mathrm{lag}}{ }^{1 / 2}$ obeys a local triangle inequality along $\gamma$ provided $\kappa^{\text {lag }}>0$; it obeys a local reverse triangle inequality along $\gamma$ provided $\kappa^{\text {lag }}<0$. To be sure, suppose $\gamma$ has the parameterization by Laguerre arc length $z\left(s^{\mathrm{lag}}\right)$. If $\Delta s_{1}^{\mathrm{lag}}, \Delta s_{2}^{\mathrm{lag}} \ll 1$, and if one ignores the terms of order $o\left(\left(\Delta s_{i}^{\mathrm{lag}}\right)^{3}\right)$, then

$$
\begin{aligned}
\sqrt[4]{12}\left(\delta_{\operatorname{lag}}\left(\mathbf{p}_{1}, \mathbf{p}_{2}\right)^{1 / 2}\right. & \left.+\delta_{\operatorname{lag}}\left(\mathbf{p}_{2}, \mathbf{p}_{3}\right)^{1 / 2}-\delta_{\operatorname{lag}}\left(\mathbf{p}_{1}, \mathbf{p}_{3}\right)^{1 / 2}\right) \\
& =-\frac{\kappa^{\lg }}{60}\left(\left(\Delta s_{1}^{\operatorname{lag}}\right)^{3}+\left(\Delta s_{2}^{\operatorname{lag}}\right)^{3}-\left(\Delta s_{1}^{\operatorname{lag}}+\Delta s_{2}^{\operatorname{lag}}\right)^{3}\right) .
\end{aligned}
$$

Since $a^{3}+b^{3}-(a+b)^{3}<0$ for $a, b>0$, it then follows that

$$
\delta_{\operatorname{lag}}\left(\mathbf{p}_{1}, \mathbf{p}_{2}\right)^{1 / 2}+\delta_{\operatorname{lag}}\left(\mathbf{p}_{2}, \mathbf{p}_{3}\right)^{1 / 2} \gtrless \delta_{\operatorname{lag}}\left(\mathbf{p}_{1}, \mathbf{p}_{3}\right)^{1 / 2}
$$


provided $\kappa^{\text {lag }} \gtrless 0$, as claimed.

The situation is exactly reversed for the quantity $\sqrt{6} \theta_{\operatorname{lag}}{ }^{1 / 2}$.

As was apparent in $\S 5.2$, the $\sqrt{6} \theta_{\operatorname{lag}}{ }^{1 / 2}$ and $\sqrt[4]{12} \delta_{\operatorname{lag}}{ }^{1 / 2}$ isometric curves are the Laguerre cycles; these are the curves with zero Laguerre curvature.

Notice, too, that an averaging of $\sqrt{6} \theta_{\text {lag }}{ }^{1 / 2}$ and $\sqrt[4]{12} \delta_{\text {lag }}^{1 / 2}$ has the improved convergence rate,

$$
\frac{\sqrt[4]{12} \delta_{\operatorname{lag}}\left(\mathbf{p}_{i}, \mathbf{p}_{i+1}\right)^{1 / 2}+\sqrt{6} \theta_{\operatorname{lag}}\left(\mathbf{p}_{i}, \mathbf{p}_{i+1}\right)^{1 / 2}}{2}=\Delta s_{i}^{\operatorname{lag}}+o\left(\left(\Delta s_{i}^{\operatorname{lag}}\right)^{3}\right),
$$

but it is not immediately clear if this is an over- or under-approximation.

7.5. Extremal curves. Using the definition of Laguerre curvature in $\S 7.1$, we here verify that the curves introduced in $\S 4$, along with their Laguerre images, are in fact the only curves with constant Laguerre curvature. We look for curves $z=x+j y$ parameterized by Laguerre arc length.

We begin by solving the following equation for $f=x^{\prime \prime} / x^{\prime}$, where the primes indicate derivatives with respect to $s^{\text {lag: }}$

$$
\left\{x ; s^{\operatorname{lag}}\right\}=\left(\frac{x^{\prime \prime}}{x^{\prime}}\right)^{\prime}-\frac{1}{2}\left(\frac{x^{\prime \prime}}{x^{\prime}}\right)^{2}=f^{\prime}-\frac{1}{2} f^{2}=\kappa^{\operatorname{lag}} .
$$

Ignoring translations in the Laguerre arc length parameter, this gives the cases

$$
f\left(s^{\operatorname{lag}}\right)=\left\{\begin{array}{l}
\sqrt{2 \kappa^{\operatorname{lag}}} \tan \left[s^{\operatorname{lag}} \sqrt{\kappa^{\operatorname{lag} / 2}}\right] \\
0 \\
-\sqrt{-2 \kappa^{\operatorname{lag}}} \tanh \left[s^{\operatorname{lag}} \sqrt{-\kappa^{\operatorname{lag} / 2}}\right]
\end{array}\right.
$$

corresponding with $\kappa^{\text {lag }}>0,=0$, or $<0$, respectively. Upon solving for $x$, this gives up to translation and dilation in the dual plane,

$$
x\left(s^{\operatorname{lag}}\right)=\left\{\begin{array}{l}
\tan \left[s^{\operatorname{lag}} \sqrt{\kappa^{\operatorname{lag} / 2}}\right] \\
s^{\operatorname{lag}} \\
\tanh \left[s^{\operatorname{lag}} \sqrt{\left(-\kappa^{\operatorname{lag}} / 2\right)}\right] .
\end{array}\right.
$$

(For instance, if $\kappa^{\text {lag }}>0$, the general solution is $x\left(s^{\operatorname{lag}}\right)=c_{1} \tan \left(s^{\operatorname{lag}} \sqrt{\kappa^{\operatorname{lag}} / 2}\right)+c_{2}$ for $c_{1}, c_{2} \in \mathbb{R}$; but the transformation $\mu=\left(z-c_{2}\right) / c_{1}$ yields the simpler form.) With these solutions, one finds that solving $d^{3} y / d x^{3}=\epsilon /\left(x^{\prime}\right)^{2}$ means solving

$$
\frac{d^{3} y}{d x^{3}}=\left\{\begin{array}{l}
+\left(2 \epsilon / \kappa^{\operatorname{lag}}\right)\left(1+x^{2}\right)^{-2} \\
\epsilon \\
-\left(2 \epsilon / \kappa^{\operatorname{lag}}\right)\left(1-x^{2}\right)^{-2}
\end{array}\right.
$$

Integrating three times gives

$$
y=\left\{\begin{array}{l}
\left(c_{1}+c_{2} x+c_{3} x^{2}\right)+\epsilon /\left(2 \kappa^{\operatorname{lag}}\right) \cdot\left(1+x^{2}\right) \tan ^{-1} x \\
\left(c_{1}+c_{2} x+c_{3} x^{2}\right)+\epsilon x^{3} / 6 \\
\left(c_{1}+c_{2} x+c_{3} x^{2}\right)+\epsilon /\left(2 \kappa^{\operatorname{lag}}\right) \cdot\left(1-x^{2}\right) \tanh ^{-1} x
\end{array}\right.
$$

for constants $c_{1}, c_{2}, c_{3} \in \mathbb{R}$. By using the remaining Laguerre transformations we can make $c_{1}=0=c_{2}=c_{3}$. In particular, a translation $z \rightarrow z+j y_{0}$ can be used to make $c_{1}=0$; a rotation $z \rightarrow\left(1+j y_{0}\right) z$ can be used to make $c_{2}=0$; and a transformation $z \rightarrow z /\left(j y_{0} z+1\right)$ to make $c_{3}=0$. We have then demonstrated that the curves in (7), (8), and (9), along with their Laguerre images, are the only curves that have constant Laguerre curvature. 
8. General curves. To conclude, we briefly outline an approach for extending the results of earlier sections to curves that are less than three-times differentiable. For instance, curves whose osculating parabolas are non-intersecting always have an invariant arc length given by (ii) and (iii) in Proposition 1. Such curves include the ones for which the concavity $d^{2} y / d x^{2}$ is a monotone (possibly discontinuous) function. At points of discontinuity, one must just include in the family of osculating parabolas a continuum of tangent parabolas whose concavities span the interval determined by the left and right limits of $d^{2} y / d x^{2}$.

It is possible to identify this class of curves with a class of positive Borel measures, and by using measure decompositions, to then determine an integrated invariant arc length that generalizes definitions (i) and (iv) in Proposition 1. The proof of the fact that the four formulations are equivalent involves Cauchy-Schwarz estimates as used in the proof of Theorems 1 and 2 and a semicontinuity argument as used by Ludwig [11]. The authors are preparing a longer work which will examine this construction for Laguerre geometry in parallel with a number of other geometries including the affine geometry of Blaschke [3, 19].

\section{Notes.}

- $\S 1-\S 2$ : A comprehensive account of Laguerre geometry in the dual plane, including the correspondence with the geometry of oriented circles and lines in the plane, can be found in Yaglom [21].

- $\S 2$ : We follow Maeda's terminology for the distinction between the direct Laguerre and the indirect Laguerre transformations [13, p.390].

- $\S 3$ : The distance $\delta_{\text {lag }}$ is completely analogous to the Coxeter distance between nonintersecting circles in the complex plane. See [6]. The distance $\theta_{\text {lag }}$ is analogous to the Kerzman-Stein distance between intersecting circles as used in $[1]$.

- §3: There is general agreement about the definition of Laguerre arc length. In particular, our definition agrees with that of Maeda. See [13, p.391].

- §3: Maeda's work [13] seems to be the most comprehensive treatment of differential Laguerre geometry in the dual plane. His work builds on earlier work of Blaschke [2], Liebmann [10], and Kubota [8, 9]. See also Maeda [12].

- §4: The normalized stationary curves also appear in Maeda [13, p.402-403]. The difference is that in our definition, Laguerre curvature is one-half the Laguerre curvature that Maeda uses. The choice made here keeps the presentation as close as possible to the case of Möbius invariant geometry over $\mathbb{C}$, where there is general agreement on constants.

- $\$ 4$ : The stationary curves of positive and negative Laguerre curvature are the dual plane realizations of an involute of a circle and a tractrix, respectively. See Maeda [13, p.402-403].

- $\S 5$ : Liebmann was the first to prove that the curves that are stationary with respect to Laguerre arc length are the curves with constant Laguerre curvature. To do this he uses the calculus of variations [10]. Maeda proved this result for finite curves by using a method designed for geometries based on linear fractional transformation groups [13, p.577-581].

- §6: The problem of finding Möbius invariant estimates for the Cauchy transform in $\mathbb{C}$ was pursued further in [1]. 
- $\S 7$ : The general formula for Laguerre curvature (15) can be proved in the same way as for the case of Möbius curvature in $\mathbb{C}$. See, for instance, Patterson [14, p.555].

\section{REFERENCES}

[1] D. E. Barrett And M. Bolt, Cauchy integrals and Möbius geometry of curves, Asian J. Math., 11:1 (2007), pp. 47-53.

[2] W. Blaschke, Untersuchungen über die Geometrie der Speere in der Euklidischen Ebene, Monatshefte für Math. und Physik, 21 (1910), pp. 3-60.

[3] W. Blaschke, Vorlesungen über Differentialgeometrie, II (Affine Differentialgeometrie), Springer-Verlag, Berlin, 1923.

[4] M. Bolt, Spectrum of the Kerzman-Stein operator for model domains, Integral Equations Operator Theory, 50:3 (2004), pp. 305-315.

[5] M. Bolt, Extremal properties of logarithmic spirals, Beiträge Algebra Geom., 48:2 (2007), pp. $493-520$.

[6] H. S. M. Coxeter, Inversive distance, Ann. Mat. Pura Appl. (4), 71 (1966), pp. 73-83.

[7] V. V. KISIL, Starting with the group $\mathrm{SL}_{2}(\mathbf{R})$, Notices Amer. Math. Soc., 54:11 (2007), pp. 14581465.

[8] T КивотA, Beiträge zue Inversionsgeometrie und Laguerre-Geometrie, Jap. Journ. Math., 1, 1924.

[9] T KuвотA, Beiträge zur Inversionsgeometrie, Tôhoku Sci Rep., 13, 1925.

[10] H. Liebmann, Zur Geometrie der Laguerre-Gruppe, Journal fur die reine und angewandte Mathematik, 154 (1924), pp. 15-19.

[11] M. Ludwig, On the semicontinuity of curvature integrals, Math. Nachr., 227 (2001), pp. 99108.

[12] J. Maeda, On the Laguerre-geometry of Plane Curves, Jap. J. Math., 17 (1940), pp. 1325.

[13] J. Maeda, Differential Laguerre geometry of plane curves, Jap. J. Math., 18 (1942), pp. 385581.

[14] B. Patterson, The differential invariants of inversive geometry, Amer. J. Math., 50:4 (1928), pp. $553-568$.

[15] M. Peternell, H. Pottmann, And T. Steiner, Hough transform and laguerre geometry for the recognition and reconstruction of special 3d shapes, Technical Report 100, Institute of Geometry, April 2003.

[16] M. Peternell and H. Pottmann, A Laguerre geometric approach to rational offsets, Comput. Aided Geom. Design, 15:3 (1998), pp. 223-249.

[17] J. Pierpont, The history of mathematics in the nineteenth century, Bull. Amer. Math. Soc., 11:3 (1904), pp. 136-159.

[18] H. Pottmann And M. Peternell, Applications of Laguerre geometry in CAGD, Comput. Aided Geom. Design, 15:2 (1998), pp. 165-186.

[19] B. C. Su, Affine differential geometry, Science Press, Beijing, 1983.

[20] K. Sushkoff, Applications of Minkowski Actions in CAGD Through Laguerre Geometry, Honors thesis, Harvey Mudd College, 2003. Available at http://www.math.hmc.edu/ seniorthesis/archives/2003/ksushkoff/.

[21] I. M. Yaglom, Complex numbers in geometry, translated from the Russian by Eric J. F. Primrose. Academic Press, New York, 1968. 
D. E. BARRETT AND M. BOLT 